Research Article

\title{
A Construction Method of Intelligent Manufacturing System under Industry 4.0 Model
}

\author{
Yue Xiao $\mathbb{D}$ and Zhiqing Zeng \\ School of Mechanical Engineering, Nanchang Institute of Technology, Nanchang, Jiangxi 330099, China \\ Correspondence should be addressed to Yue Xiao; xy@nit.edu.cn
}

Received 8 October 2021; Revised 25 October 2021; Accepted 26 October 2021; Published 9 November 2021

Academic Editor: Tongguang $\mathrm{Ni}$

Copyright (c) 2021 Yue Xiao and Zhiqing Zeng. This is an open access article distributed under the Creative Commons Attribution License, which permits unrestricted use, distribution, and reproduction in any medium, provided the original work is properly cited.

\begin{abstract}
Starting from the current problems facing Industry 4.0, this article analyzes the changes in the macro and industrial environment that Industry 4.0 faces and explains the problems, opportunities, and strategies for the manufacturing industry in the external environment. First, the reference system of the intelligent manufacturing system, the current status, and the existing problems of industrial production management are analyzed through the investigation of the status quo of industrial production and management. This puts forward the detailed requirements of the industrial intelligent manufacturing system in the data acquisition layer, data storage layer, and analysis and decision support layer and then designs the hierarchical structure of the industrial intelligent manufacturing system. Subsequently, it adopts design methods and lists product manufacturing costs, pointing out that Industry 4.0 requires industrial transformation, and finally proposes the strategic direction of smart manufacturing in combination with the Industry 4.0 network strategy. At the same time, in view of the problems of long parameter measurement time and untimely system feedback in the existing koji-making process, an online parameter measurement method based on network optimization is proposed. On the basis of the neural network, an industrial neural network with double hidden layers and self-feedback of the output layer is proposed. Through algorithm comparison experiments, the proposed parameter prediction model based on industrial neural network has better prediction results and higher accuracy. Finally, a comparison of cost, quality, delivery time, etc., before and after the implementation of Industry 4.0 intelligent manufacturing is carried out. An intelligent solution is proposed, the implementation goal is formulated, and the implementation is gradually implemented in stages, and finally an intelligent upgrade and transformation are realized. It is shown in many aspects that intelligent manufacturing provides a powerful means for enterprises to achieve agility, virtualization, lean, integration, and collaboration, and it can bring efficiency, reliability, and safety to the manufacturing process of enterprises.
\end{abstract}

\section{Introduction}

With the gradual rise of customized manufacturing, intelligent manufacturing systems have been widely used, and the resulting intelligent scheduling problems have become a hot research topic [1]. Industry 4.0 focuses on intelligent manufacturing and strives to ensure that the manufacturing industry can continue to develop at a high speed with green, environmental protection, intelligence, and efficiency. The three parts of smart factory, smart production, and smart logistics form the core of Industry 4.0 [2]. Regardless of whether it is smart production or smart logistics, it requires systematic and efficient manufacturing, intelligent allocation of manufacturing resources, and reasonable management of warehousing logistics. These are all multiobjective intelligent scheduling problems. Intelligent scheduling plays a role equivalent to the human brain in intelligent manufacturing, and system modeling and intelligent scheduling are the basis of intelligent manufacturing systems [3]. In particular, the introduction of Industry 4.0 makes intelligent scheduling play the role of the core brain in both smart factories and smart logistics in smart manufacturing, which is the basis of smart manufacturing [4]. Most of the intelligent scheduling problems are combinatorial optimization problems, and their computational theory has been proven to be NP-hard problems. The traditional method of studying combinatorial optimization cannot find the optimal solution of the scheduling 
problem in polynomial time, and then people began to study to find the near-optimal solution in polynomial time to meet the demand of production scheduling [5].

The purpose of intelligent scheduling is to complete the production tasks of production processing and machine assembly under the premise of satisfying various constraints of the manufacturing system. In the process of intelligent production, due to the frequent changes of production goals, such as the acceleration of product upgrades, the intelligent manufacturing system must also be adjusted according to the new production tasks to ensure that the machine can complete the production tasks with a higher utilization rate [6]. More importantly, in the process of intelligent manufacturing, production is fully automated and unmanned. Once an unreasonable processing arrangement occurs, the machine may collide and cause a safety accident, or two robots may compete for one processing equipment for conflict of loading materials [7]. Therefore, intelligent scheduling plays an extremely important role in the processing safety, equipment utilization, processing cost, and task completion of the intelligent manufacturing system. From the perspective of the manufacturing system, when the number of workpieces manufactured is small or the product is constantly changing, intelligent scheduling is irreplaceable in intelligent production. Intelligent manufacturing system has unique advantages in solving the above aspects [8-10]. And intelligent scheduling is the key to ensuring efficient, reliable, and safe production of intelligent manufacturing systems, so the research on intelligent scheduling has very important practical significance.

With regard to the modeling of intelligent manufacturing system, this paper studies how to use the industrial network to model and analyze the intelligent manufacturing system and proposes the modeling idea from the basic manufacturing unit to the entire manufacturing system. For the research on system model deadlock, we analyze the reachability graph of industrial network system to judge the deadlock situation during system operation and use the established model to study the scheduling method of single task and multitask. In the process of researching batch scheduling, this paper puts forward the idea of combining genetic algorithm and simulated annealing algorithm to form a hybrid algorithm applied to the field of intelligent scheduling. The inspection and evaluation stage mainly analyzes the current situation of K company's internal management and external competition environment and determines the research objectives, planning principles, implementation methods, evaluation index system, and technical methods. Research on the application of Internet of Things technology, industrial robot technology, cloud computing and big data technology in solid wood customization enterprises integrates ERP system, SCM system, CRM system, PLC system, MES system; realizes the interconnection of data and information; and cooperates with each other, promoting each other to form a complete intelligent manufacturing system.

\section{Related Work}

This article mainly focuses on the research of Industry 4.0 application systems. The research of Industry 4.0 for specific applications mainly focuses on transportation, medical treatment, smart grid, building, and environmental detection. We studied the design method of the transportation-oriented Industry 4.0 system and conducted in-depth research on several key system technologies, such as vehicle perception technology, wireless network protocol, multisensing node information fusion algorithm, and vehicle information feature extraction, and finally realized the transportation Industry 4.0-oriented Vehicle perception and recognition system [11].

Marques et al. [12] looked forward to the application of Industry 4.0 systems in smart medical care and proposed the general development trend of real-time sensing, adaptive response, and remote control medical services. They use sensors installed on the patient to sense vital signs, such as heartbeat and blood pressure. This information can be sent to medical caregivers on a regular basis so that they can provide real-time and effective care to patients. Annanth et al. [13] proposed the microgrid Industry 4.0 system architecture, introduced Industry 4.0 technology into the construction of the microgrid, and gave the microgrid Industry 4.0 architecture model. On this basis, the microgrid Industry 4.0 physical layer, adjustment layer, coordination layer, 6-layer system framework of connection layer, network layer, and application layer were proposed. $\mathrm{Hu}$ and Gao [14] researched and designed the building structure health monitoring Industry 4.0 system and studied how to build a reusable architecture so that physical testing and computer simulation testing can be precisely combined, which can effectively support mixed testing of building structures. Wang et al. [15] studied the use of wireless sensor networks (WSN) to construct distributed building structure health monitoring and defect location methods. The paper points out that the existing centralized sensor network structure health monitoring system has problems, such as data collection delay and large network energy overhead, and proposes a distributed processing method. Most of the research in the abovementioned literature is still at the stage of theoretical research on Industry 4.0 architecture, and there is little experience in combining theory with practice. This article will focus on the application of Industry 4.0 theoretical architecture to the design of specific intelligent manufacturing systems.

Tao et al. [16] proposed to combine cloud computing and next-generation Internet technology to study the semantic middleware of Industry 4.0. He Ming et al. [10] studied the Industry 4.0 architecture from the perspectives of system, function, and technology. It also gives the research direction of the Industry 4.0 system and the current challenges. At the same time, a three-tier architecture of Industry 4.0 physical layer, network layer, and application layer was constructed, and related concepts were described and analyzed in depth. Finally, the system architecture was applied to intelligent transportation, which proved the practicality of the architecture. Some scholars have proposed a service-oriented Industry 4.0 architecture, which is divided into a node layer, a network layer, a resource layer, and a service layer. Indepth discussion on the functional modules and technical foundations of each layer is also given, and the future research direction of the Industry 4.0 system is also given. At 
the same time, it is pointed out that intelligent manufacturing technology is a highly flexible and highly integrated way, through computer simulation of human brain analysis, judgment, and decision-making [17, 18]. Unlike the computer integrated manufacturing system that emphasizes the integration of enterprise material flow and information flow, the intelligent manufacturing system emphasizes the self-organization ability of the entire manufacturing process. It is believed that the intelligent manufacturing system is a combination of intelligence, integration, and automation, with super self-learning ability and self-organizing ability. Some scholars also believe that intelligent manufacturing systems should have self-discipline, self-organization, self-learning, and self-optimization capabilities. Second, from the perspective of industrial applications, we research the development of the intelligent part of the entire manufacturing environment and improve the intelligent manufacturing technology and manufacturing system [19-21].

\section{Model Construction of Intelligent Manufacturing System Based on Industry 4.0 Model}

3.1. Industry 4.0 Level Analysis. Industry 4.0 is the industrial development strategy of the fourth industrial revolution with intelligent manufacturing as the core. The strategy aims to transform manufacturing into intelligent manufacturing by making full use of information and communication technology and cyberspace virtual systems, a combination of information and physical systems. The main way to achieve this is to fully control the entire process from product demand to manufacturing by building a cyber-physical fusion system, thereby realizing efficient production process management. The Industry 4.0 system is mainly composed of a computing system, a communication system, and a control system, forming the $3 \mathrm{C}$ model of the Industry 4.0 system. Figure 1 shows the Industry 4.0 hierarchical topology.

Among them, a large number of sensor nodes and actuator nodes are distributed in the physical world, complete the real-time perception of the physical world, and send them to the computing system and control system through the communication network. The computing system completes various computing tasks required by Industry 4.0 and provides various information services including data mining, statistics, and forecasting.

$$
Y(x)=\sum_{x=1, y=1}^{n} p(y \mid x) \times f(y \mid x) .
$$

The control system uses the data obtained from the analysis of the perception network and the computing system to perform scientific operations on the objects in the physical world, thereby realizing feedback control of the physical world. The communication network of the Industry 4.0 system can be seen as an extension of the sensor network, a combined communication network composed of sensor networks, computer networks, and next-generation communication technologies.

$$
\begin{aligned}
& g(x)-\sum_{i, j=1}^{n}[s(1, i)+s(2, i)+\cdots+s(j, i)] / s(i, j)=0, \\
& \frac{p^{\prime}(x)-p(x)}{p(x)}-\int \frac{\partial f(x)}{\partial x} \mathrm{~d} x=0 .
\end{aligned}
$$

The activation function of the network has two main functions: first, it can provide a filtering function for the input of the unit node. It is generally hoped that when the correct input is given, the unit is in an active state, near +1 . On the contrary, when the wrong input is given, the unit is in an inactive state, near 0 ; second, no excitation function is applied, and the transfer between neural network layers is just a linear transformation, which leads to insufficient expressive ability of the network model, and the excitation function is the fact that nonlinear factors can be introduced to increase the expressive ability of the model.

$$
\begin{aligned}
& \{\sigma(x, x), \sigma(y, y), \sigma(z, z)\} \longrightarrow\{\varepsilon(x, y), \varepsilon(y, z), \varepsilon(z, x)\}, \\
& U=1 / 2 \times \int_{\Omega} \sigma(z, x) \sigma(x, y) \sigma(y, z) d \Omega .
\end{aligned}
$$

During the production process, changes in market demand and raw material supply can be changed and adjusted in time. More importantly, through the intelligent assistance system, employees are relieved. Their work is not on a stepby-step basis, but more energy is invested in innovation to make their work more valuable. This new model is conducive to employees to better integrate their own work, improve work efficiency, and increase work value.

\subsection{Construction of Intelligent Manufacturing Platform.} Intelligent manufacturing is the integration of manufacturing technology with intelligent technology, digital technology, and network technology in the entire life cycle of product design, manufacturing, operation management and after-sales service, intelligent reasoning, and intelligent decision-making and control to achieve timely response to consumer needs. It is the general term for product design, manufacturing, and supply chain logistics. It is divided into four types: intelligent production, intelligent design, intelligent manufacturing, and intelligent management. The characteristics of intelligent manufacturing are as follows: intelligently perceive and acquire production personnel, production equipment, raw and auxiliary materials, manufacturing environment, and related information in the manufacturing process, interconnection, collaboration, analysis and statistics, forecasting and early warning, decision support, control and execution. In smart manufacturing, the MES system based on smart production is the core component of smart manufacturing.

Figure 2 is the result of dispatching layer allocation of the intelligent manufacturing platform. In the planning phase, the planning and dispatching layer provides material 


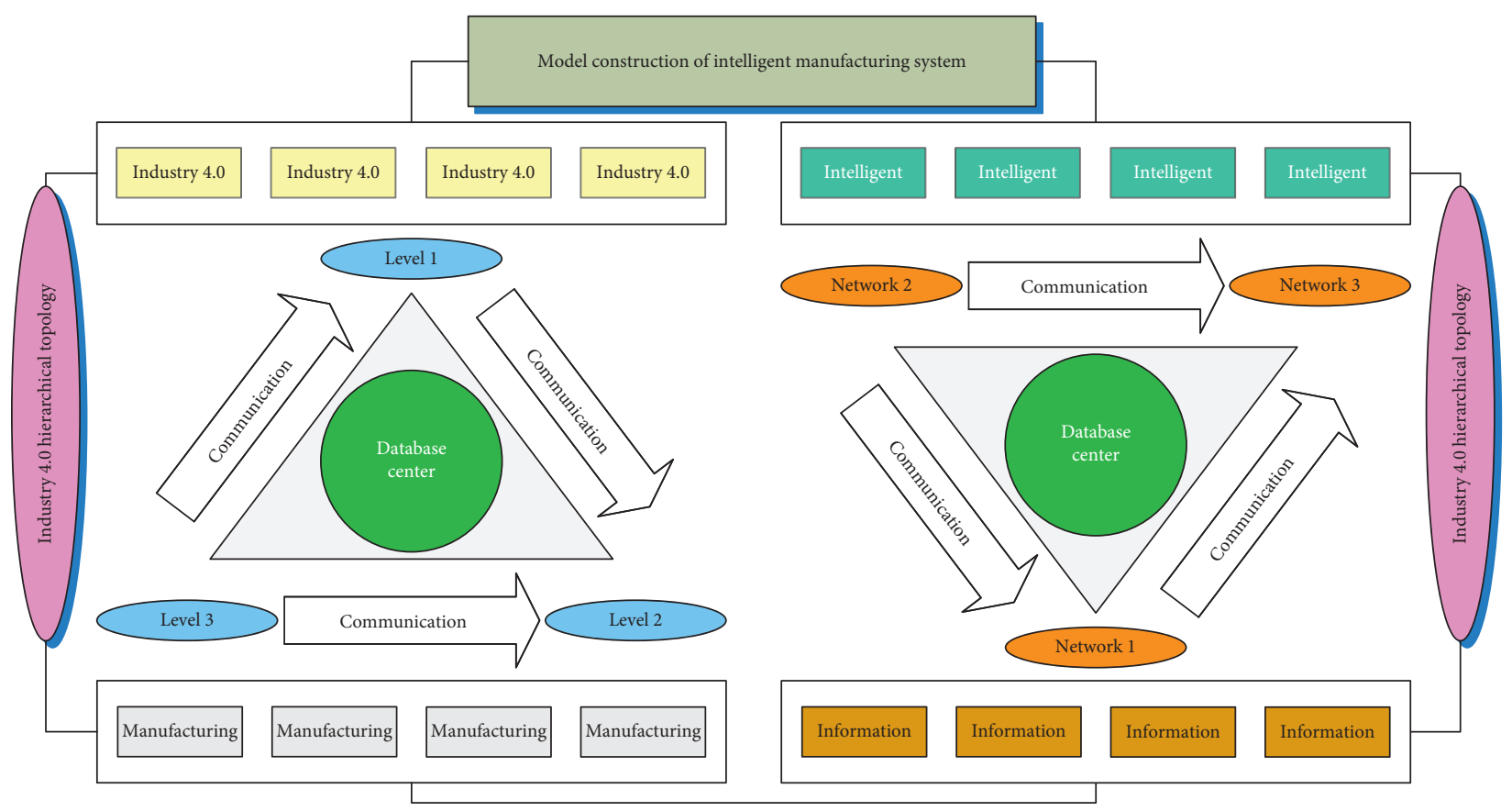

Figure 1: Industry 4.0 hierarchical topology.

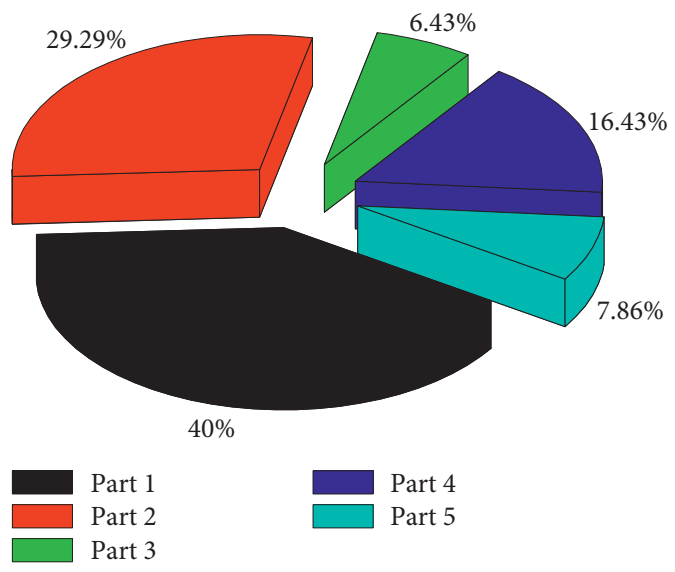

FIGURE 2: Distribution results of the scheduling layer of the intelligent manufacturing platform.

demand plans to the raw material depots and distribution centers; before production, the execution tracking layer sends out preparation instructions to the raw material depots and distribution centers; during production, the execution tracking layer sends the raw material depots and the control system of the distribution center issues an outbound instruction. The three-level coordination can ensure the timely and accurate supply of materials and ensure on-site production. In the production of refrigerators, the matching of cabinet doors is a difficult problem. Through three-level coordination, the matching of cabinet doors can be effectively achieved. When the planning and scheduling layer issues instructions, the execution tracking layer then issues corresponding process control parameters to the process control layer according to the instructions. At the same time, the process control layer feeds back information to the execution tracking layer, and the execution tracking layer updates the execution status of the work order and feeds it back to the planning and scheduling layer. Through the "three-level linkage," the correctness of the discharge of the library can be checked, and the error-proof interlocking of the execution of the work order can be performed. The basic requirements of automated intelligent manufacturing, data, intelligent equipment, and networks are all prepared to achieve this goal. The data in the entire production process is intelligently collected and dynamically produced to reflect the operation of the entire system in real time. According to the data in the knowledge base, the equipment is automatically maintained on a periodic basis, self-diagnosed, and automatically repaired to avoid the occurrence of failures. Not only the production equipment, but also the overall monitoring of the operation of the entire intelligent system, automatically adjust the production line, realize different production, analyze the results, and predict and prevent systemic failures. We realize interconnection and humanmachine integration; integrate resources to quickly respond to market demand; realize intelligent, visual, and fully automated production: extract effective and provide a basis for top-level decision-making; reduce production costs, improve production efficiency, improve product quality, and shorten products cycle.

3.3. Model Weight Factor Update. At the system level, the intelligent manufacturing system is highly integrated with multiple subsystems. It is an organic whole, supplemented by each other, and interconnected with data to jointly achieve the goal of intelligence. At the control level, PLC controls intelligent equipment, system scheduling management, production execution system executes automated 
tasks, allocates orders, drives equipment, and collaborates to complete the production process. On the execution layer, the execution layer is the real-time perception object of the control layer and the actual terminal monitored by the system layer. Various on-site equipment, materials, areas, lines, etc., use RFlD technology to enable any entity in the system to have a unique identity. The sensing system collects equipment data in the production logistics process in real time and uses big data analysis technology to control the operation of the equipment in real-time parameter. The equipment layer is the entire intelligent equipment, various auxiliary units, which are indispensable for the production site.

Figure 3 is a ladder diagram of the weight of the manufacturing system data supply chain. The internal supply chain communicates with the external supply chain daily to ensure the smooth progress of product production. In addition, the supply chain is integrated to a certain extent through ERP, MES, and other information management systems and through the perception layer (RFID, barcode, GPS, etc.). The network layer (private network, Internet, wired and wireless communication network, etc.) and the application layer (PC, PAD, mobile phone, etc.) seamlessly connect the supply chain to achieve real-time monitoring of the customization process and problem solving appear to give intelligent judgments and make timely responses. Therefore, the corresponding supply chain is basically like a pull supply chain, which drives the production of the workshop by the customer's demand for enterprise products, but it is not a complete pull supply chain model because the design of customized products is not complete. This will involve the MES system and the EI punching system. As the manufacturing execution system, MES is in the middle part, as an intermediate connecting the upper-level planning system and the bottom-level control system. Its main task is the "real-time" production and scheduling of the workshop production process. For nonstandardized and nonmodular production, some parts are often fixed into standard modules for inventory storage through analysis and statistics. When a customer's order contains these modules, they can be used directly, thereby saving parts. The processing time has shortened the delivery time of the order to a certain extent, which not only improves the production efficiency, but also ensures the economic benefits of the enterprise.

3.4. Model Scheduler Design. Manufacturers use the "cyber-physical system" as the framework to build a global industrial network of factory equipment, warehouse management, and industrial products. In the manufacturing sector, Industry 4.0 means that all equipment of an enterprise can independently exchange information. Industry 4.0 is a small smart machine, a small storage system, and efficient product equipment that realize operation and mutual control. Through Industry 4.0, various industrial processes such as manufacturing, engineering, supply chain, and life cycle management are further linked together, and product production is carried out in a brand-new way. When the production instruction reaches the system composed of Industry 4.0, it can flow through the business processes of factories and companies, and the interconnection of equipment can create a derived equipment and product value system, forming a network of manufacturing systems through the Internet of Things. The idea of the Industry 4.0 system includes the whole process of how the physical world information is perceived by the information world in real time and how the information world calculates and makes decisions on the collected data. The ultimate goal of the Industry 4.0 system is to use the existing physical world infrastructure (including perception, computing, and communication hardware and software facilities) to realize intelligent monitoring from the physical world to the information world and from the information world to the physical world to the extent that the information world and the physical world are completely integrated.

The Industry 4.0 system architecture includes three systems: computing system, communication system, and control system. Figure 4 shows the design process of the model scheduler. Among them, the computing system is the central processor of the Industry 4.0 system, responsible for completing all the calculations and decision-making processes of the Industry 4.0 system. At the same time, it can bear the pressure of analysis and processing of massive data and perform fusion processing on multiple data; the communication system is the network transmission part of the Industry 4.0 system, which is responsible for completing the communication and data transmission between devices, from sensors, actuators to information. MES decomposes and refines the production plan information from ERP software to form specific operating instructions. The central network is interconnected; the control system is the central nerve of the Industry 4.0 system, which can perceive all the information in the physical world and respond to the information. Through the control system, the system can be operated according to predetermined rules, and the operation of the system can be maintained or changed. From the perspective of the system view, the Industry 4.0 system is relatively broad and has little guiding significance in practice.

\section{Application and Analysis of Intelligent Manufacturing System Model Based on Industry 4.0 Model}

4.1. Industry 4.0 Data Preprocessing. The data set mainly includes a training set and a test set. The training set is used to adjust the network connection weight and other internal structures, and the test set is used to verify the results of network training. The training set and test set of the experimental data are from the measured data of the product instrument during the process of making music. At present, 70 sets of data are randomly selected to construct the data set (product parameters are affected by factors such as seasons, and the data set is selected from the measured data in the past two months), of which 60 sets of data are used as the neural network training set, and 10 


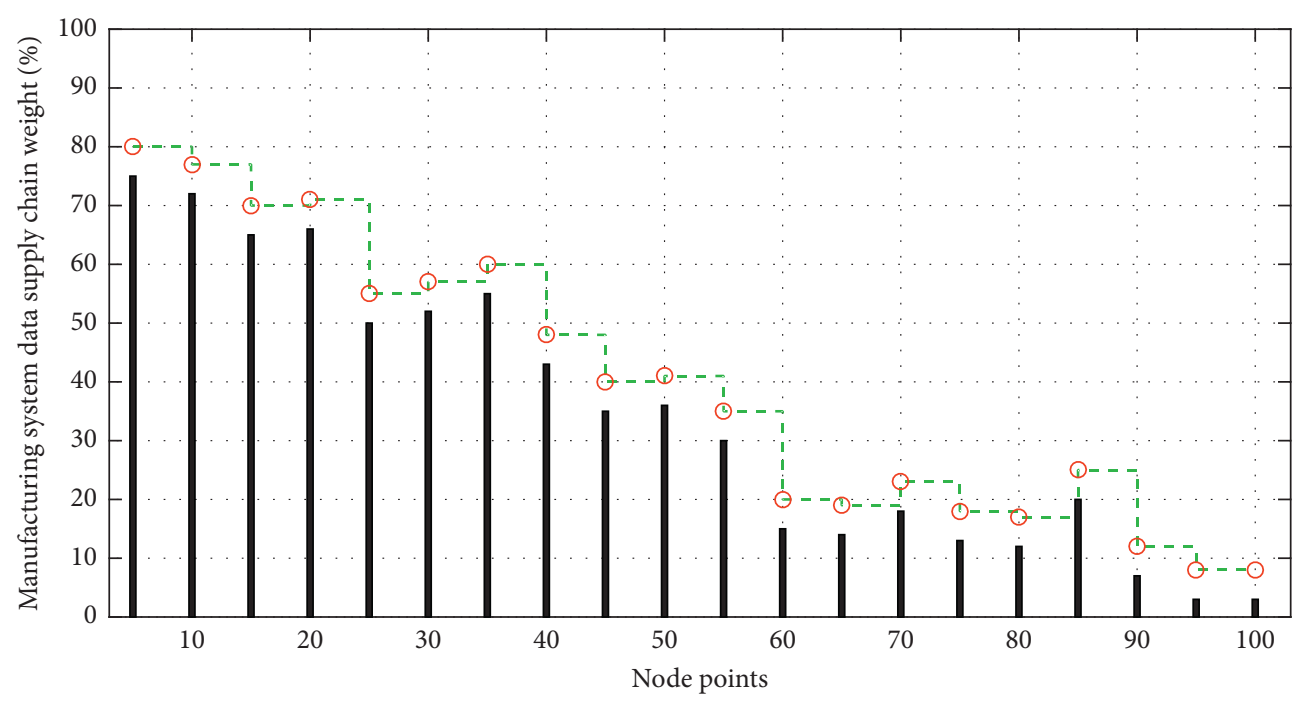

Figure 3: The ladder diagram of the weight of the manufacturing system data supply chain.

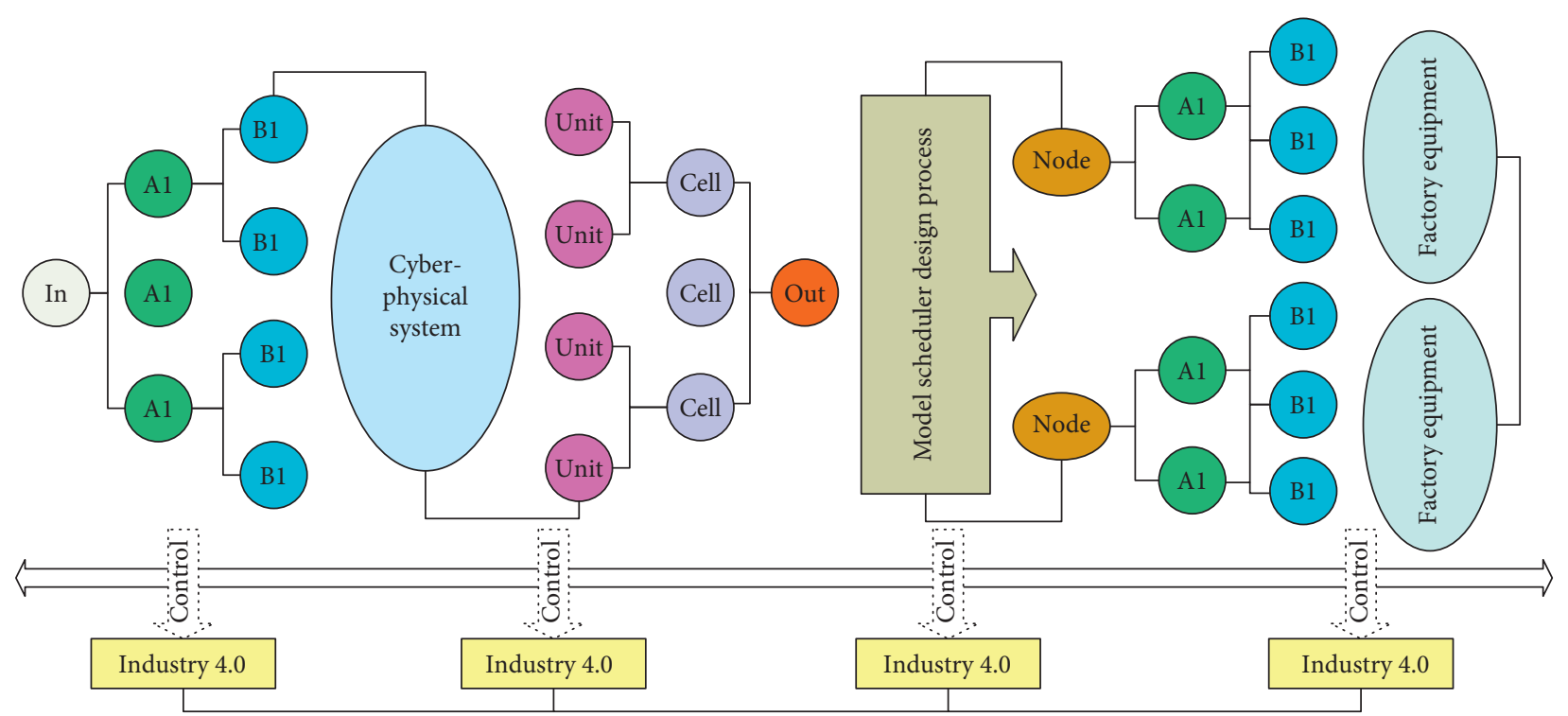

FIgURE 4: Model scheduler design process.

sets of data are used as the algorithm test set. For an example of a scheduling problem, the iterative curves of the optimal solutions of the three algorithms are given. GA-HS1 did not obtain a feasible solution to the problem in the initial iteration stage, so it is not drawn. It can be seen that the order preallocation strategy and the chromosome adjustment link can increase the probability of obtaining a feasible solution so that the algorithm can search in the entire feasible region, and a better solution can be searched in a limited time; that is, GA-HS has better convergence performance. Figure 5 is a histogram of the data convergence effect of the manufacturing system.

According to the basic method of fuzzy industrial network, the network elements of warehouses, tokens, and changes are defined, the topology structure of the network system, namely, the network relationship, is established, and the constraint conditions of the dynamic behavior of the system are given according to the actual production

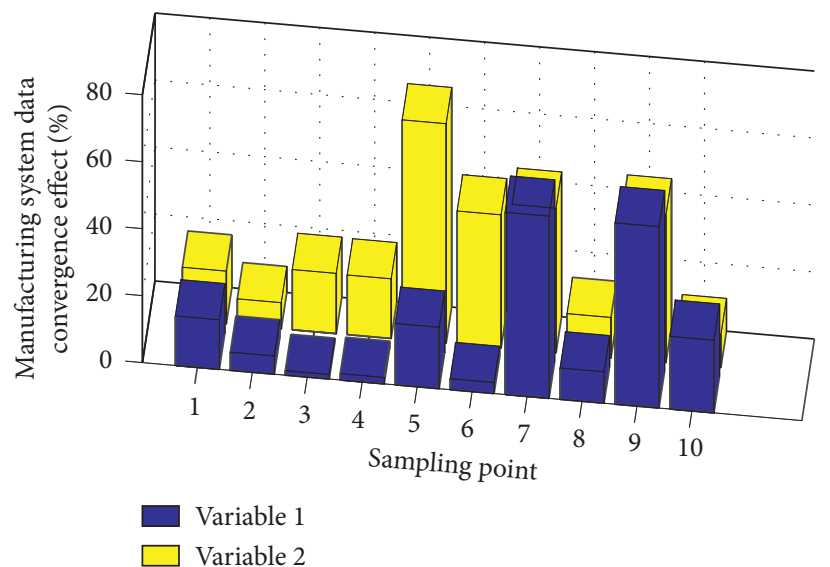

FIGURE 5: Histogram of data convergence effect of manufacturing system. 
conditions and production requirements with a fuzzy industrial network energy consumption model suitable for aluminum alloy casting production process. The model describes the main production process and the energy conversion process, that is, the energy consumption process. The dynamic behavior of the system energy consumption is expressed as stimulating continuous changes, and the result of the behavior, the change of the system state, is described by the continuous stock token number, which is the label. The model simulates the relationship between the change in process status and energy consumption process caused by equipment actions derived from operating instructions. Taking into account the interdependence of multiobjectives and decision variables, the aluminum casting decisionmaking process is divided into two layers. The first layer uses a genetic algorithm based on a mixed code of real numbers and integers to select the corresponding furnace group or furnace group set for each order. The material data of the order provides timely feedback to the EI heart system, and the production plan is effectively connected with the ERP procurement plan and realizes the material analysis of the order, the analysis of the process route and the optimization of the production plan: make a refined production schedule plan for the production order, establish production instructions, and assign the production task list to the corresponding equipment; perform job scheduling, monitor the production progress of the workshop, collect process data, quality data, time data, and other big workshop data, accurately obtain the dynamic data of the workshop; realize order matching and warehouse management; generate various production analysis report; and feedback equipment related information.

\subsection{Realization of Intelligent Manufacturing Simulation.} Product instrument detection is a continuous measurement process, and data is uploaded every 5 seconds on average. A measurement process takes about 22 minutes, and approximately 260 data are generated. This article uses neural network to predict the product. The data node selects one point every minute and selects the measurement data of the first 5 minutes. At the same time, the quality of the test sample is used as an influencing factor. Then, there are 6 input nodes, and the output value is the measured value of the final wheat product. We select the measured value, sample quality, and final product 5 minutes before the measurement process for network training. Based on the overall structure of the model established above, using the working mechanism of each device in the product group and the actual structure and parameters of the system, the aluminum alloy melting and casting energy consumption model is solidly modeled, and the operating parameter settings under the actual working conditions of the production are used. We set value and operation behavior, equipment action, simulate the dynamic behavior of product group, and compare and verify the simulation result with the actual temperature recorded during the production.

Figure 6 is a line graph of the data training efficiency of the manufacturing system. It can be seen that the deviation

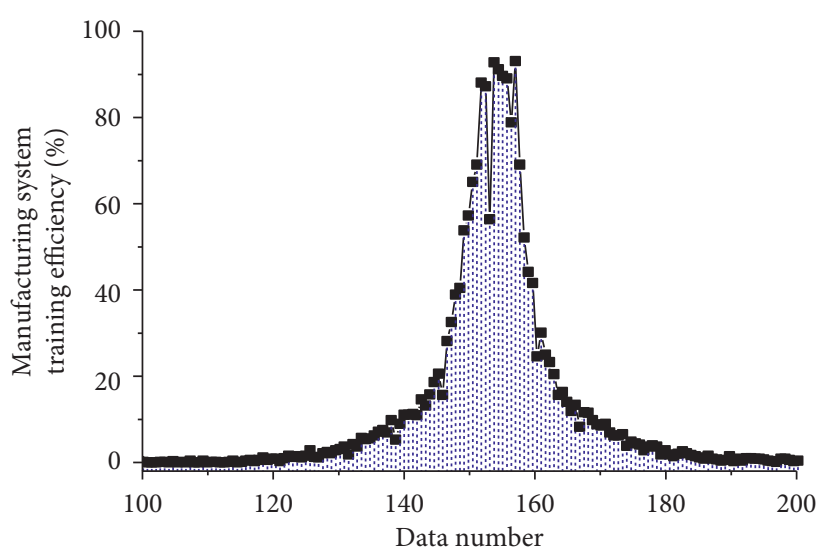

FIGURE 6: Line graph of data training efficiency of manufacturing system.

of the prediction results of the industrial neural network in 10 experiments is generally small, and the error is the smallest in 7 of the 10 experiments. It can be seen that the overall prediction accuracy of the algorithm is high. In the figure, it can be seen that the minimum cumulative error of the industrial algorithm for 10 experiments is $1.84 \%$, and the average error is only $0.184 \%$, which is far lower than the actual production $0.5 \%$ measurement accuracy requirement, which can meet the on-site measurement accuracy requirement. The fluctuation curve of the predicted value and the measured value can further illustrate that among the three algorithms, the industrial forecast result is the closest to the measured result, and the forecast error of the industrial algorithm is generally lower than that of the other two algorithms, and the forecast deviation is basically below $0.3 \%$. A small number of prediction errors are large, but all are below $0.5 \%$, which meets the actual production accuracy requirements. The fluctuation curve of the predicted value and the measured value can further illustrate that, among the three algorithms, the industrial forecast result is the closest to the measured result, and the forecast error of the industrial algorithm is generally lower than that of the other two algorithms, and the forecast deviation is basically below $0.3 \%$. A small number of prediction errors are large, but all are below $0.5 \%$, which meets the actual production accuracy requirements.

4.3. Case Application and Analysis. Deploy the temperature and humidity sensor in the yeast fermentation room; each room deploys 5 sensing nodes, including 3 temperature sensing nodes inside the yeast, which are deployed diagonally inside the room, 1 ambient temperature sensing node, and 1 ambient humidity The sensing node uploads the temperature and humidity data to the system server in real time through the real-time perception of the ambient temperature and humidity and the internal temperature of the curve, so as to achieve the purpose of remote monitoring and decision-making. The settings of the three network nodes are consistent, with 6 input nodes, 8 hidden layer nodes, and 1 output node. The layer proposes a harmony 


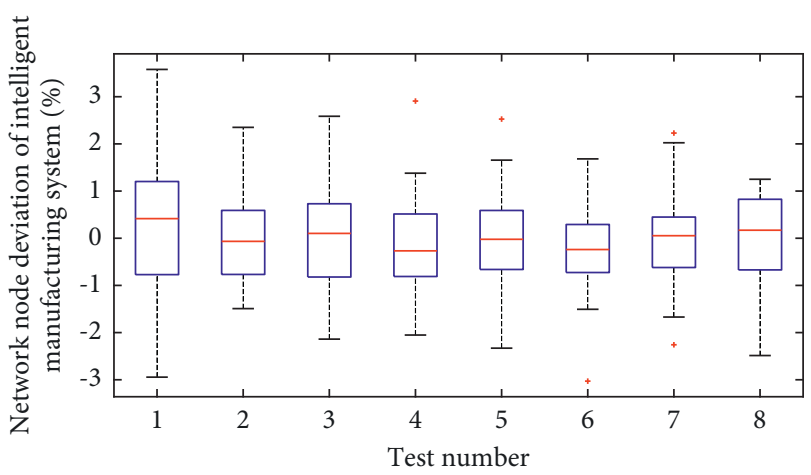

FIGURE 7: The distribution of network node deviation in intelligent manufacturing system.

search algorithm based on chaotic sequences to make decisions on the processing order of each order on each furnace group. Each layer selects the same type of state transition function. The hidden layer activation function selects the sigmoid function to improve the nonlinear approximation ability of the model. The output layer selects the linear function to reduce the deviation between the predicted value and the measured value. The convergence condition set in the algorithm experiment is that one of the network training errors mse is less than 0.005 or the number of training iterations is greater than 5000 times. Figure 7 shows the distribution of deviations in the network nodes of the intelligent manufacturing system.

We build the WCC software factory product database, enter the R\&D product modules, dock processing equipment, and complete the WCC software's order splitting function. After the dealer's order is verified and confirmed, the order is split through the WCC software, and the BOM list is exported through the E-fang auxiliary expansion interface, including the billing list, the bill of materials, the hardware list, the quotation list, the outsourcing list, and other reports. The implementation of share production management system lays a data foundation. The logo of the fuzzy place P18 represents the change trend of the molten aluminum temperature, which is represented by a solid line, the excitation of the discrete transition T6 represents the feeding action, which is represented by a dotted line, and the excitation of the discrete transition T7 represents the action of the valve, represented by a dashed line. The simulation results show that the change trend identified by the temperature library basically reflects the change trend of energy consumption during this production operation, and the excitation of each change in the model reflects the change of the working state of each device.

Figure 8 shows the distribution of preallocated values of orders in the intelligent manufacturing system. For examples of scheduling problems with scales of $10 \times 5,10 \times 10,30 \times 5$, and $30 \times 10$, the performance comparisons of each link in the proposed method are given respectively. Among them, the parts in bold are examples of the same problem, which are obtained by different methods. The best solution is GA-HS-1 no-order preallocation strategy and chromosome adjustment link and GA-HS-2 no-order preallocation strategy. It

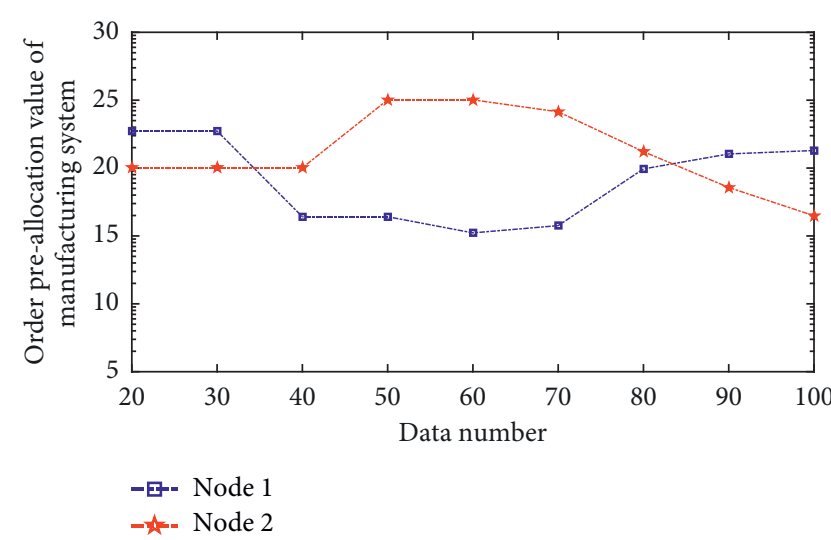

FIgURE 8: Value distribution of order preallocation in intelligent manufacturing system.

can be seen from this that for most scheduling problem instances, the methods proposed in this chapter can achieve better scheduling goals and have greater improvements. The chromosome adjustment link can increase the probability of obtaining a feasible solution, allowing the algorithm to search in the feasible area, and the order preallocation strategy can further improve the quality of the solution. For some small-scale scheduling problem examples, such as the $10 \times 5$ scheduling problem example, GA-HS- 1 has a higher probability of obtaining the best solution. This is because the order preallocation strategy artificially reduces the search space for understanding; thus, in a smaller solution space, GA-HS-1 can search the entire solution space so that the probability of obtaining the optimal solution is greater. For large-scale scheduling problem examples, such as a scheduling problem with a scale of $30 \times 10$, the order preallocation strategy can increase the probability of obtaining the optimal solution within a limited search time.

\section{Conclusion}

This paper analyzes the intelligent manufacturing system based on Industry 4.0 and divides the task types into two types of problems: fixed delivery and fuzzy delivery according to the delivery situation so that the research of scheduling algorithm is more pertinent. Particularly for the fuzzy delivery problem, through its modeling and analysis, the algorithm becomes a scheduling algorithm that is judged by the processing cost, which is closer to the actual situation, and the application range is greatly expanded. At the same time, a music making intelligent manufacturing system architecture based on Industry 4.0 (a three-tier Industry 4.0 system architecture of perception layer, network layer, and application layer) is proposed to guide the specific design and implementation of the system. We carry out the hierarchical design of the system according to the proposed Industry 4.0 system architecture. The system perception layer design proposes four types of perception node design schemes to complete the comprehensive perception of music making parameters. The system network layer proposes a heterogeneous networking model for Industry 4.0 intelligent making and uses network model simulation to verify the 
feasibility of the networking model. The system application layer puts forward the software design process of the realtime perception system based on the $\mathrm{B} / \mathrm{S}$ architecture and designs the key code of the industrial algorithm based on the Java language. The access layer realizes unified access to heterogeneous data sources and heterogeneous systems through data integration components and business integration components. Among them, the business integration component mainly realizes the integration of third-party business systems such as the upper-level ERP system, the bottom-level control system, and the supply chain system. Aiming at the shortcomings of the high coupling between traditional algorithm services and system business, the RPC algorithm service platform design is proposed, which separates the industrial neural network algorithm services and uses remote service calls to perform algorithm predictions, which can greatly improve the reliability of system software. Based on the analysis of the status quo of intelligent manufacturing in the industry, combined with the application of Internet of Things technology, industrial robots, and big data cloud computing technology in the industry, this paper formulates an implementation plan and implementation steps and verifies through examples to build an Industry-oriented 4.0 customized intelligent manufacturing theory system that has certain reference value and guiding significance for the development of intelligent manufacturing industry.

\section{Data Availability}

The data used to support the findings of this study are available from the corresponding author upon request.

\section{Conflicts of Interest}

The authors declare that they have no conflicts of interest or personal relationships that could have appeared to influence the work reported in this paper.

\section{Acknowledgments}

This study was supported by the Science and Technology Project of Education Department of Jiangxi (Grant no. GJJ151121), the National Natural Science Foundation of China (Grants nos. 52165015 and 51565037), and the Natural Science Foundation of Jiangxi (Grant no. 20181BAB206022).

\section{References}

[1] B.-h. Li, B.-c. Hou, W.-t. Yu, X.-b. Lu, and C.-w. Yang, "Applications of artificial intelligence in intelligent manufacturing: a review," Frontiers of Information Technology \& Electronic Engineering, vol. 18, no. 1, pp. 86-96, 2017.

[2] G. J. Cheng, L. T. Liu, X. J. Qiang, and Y. Liu, "Industry 4.0 development and application of intelligent manufacturing," in Proceedings of the 2016 International Conference on Information System and Artificial Intelligence (ISAI), pp. 407-410, Hong Kong, China, June 2016.

[3] S. Shan, X. Wen, Y. Wei, Z. Wang, and Y. Chen, "Intelligent manufacturing in industry 4.0: a case study of Sany heavy industry," Systems Research and Behavioral Science, vol. 37, no. 4, pp. 679-690, 2020.

[4] R. Y. Zhong, X. Xu, E. Klotz, and S. T. Newman, "Intelligent manufacturing in the context of industry 4.0: a review," Engineering, vol. 3, no. 5, pp. 616-630, 2017.

[5] C.-F. Chien, T.-y. Hong, and H.-Z. Guo, "A conceptual framework for "industry 3.5 " to empower intelligent manufacturing and case studies," Procedia Manufacturing, vol. 11, pp. 2009-2017, 2017.

[6] Q. Gao, R. Shi, and G. Wang, "Construction of intelligent manufacturing workshop based on lean management," Procedia Cirp, vol. 56, pp. 599-603, 2016.

[7] Y. Guo, N. Wang, Z. Y. Xu, and K. Wu, “The internet of things-based decision support system for information processing in intelligent manufacturing using data mining technology," Mechanical Systems and Signal Processing, vol. 142, Article ID 106630, 2020.

[8] S. Yang, J. Wang, L. Shi, Y. Tan, and F. Qiao, "Engineering management for high-end equipment intelligent manufacturing," Frontiers of Engineering Management, vol. 5, no. 4, pp. 420-450, 2018.

[9] S. Wei, J. Hu, Y. Cheng, Y. Ma, and Y. Yu, "The essential elements of intelligent manufacturing system architecture," in Proceedings of the 2017 13th IEEE Conference on Automation Science and Engineering (CASE), pp. 1006-1011, Xi'an, China, August 2017.

[10] M. He and B. Walheer, "Spillovers and path dependences in the Chinese manufacturing industry: A firm-level analysis," The Journal of Development Studies, vol. 56, no. 4, pp. 817839, 2020.

[11] S. Wang, J. Meng, Y. Xie, L. Jiang, H. Ding, and X. Shao, "Reference training system for intelligent manufacturing talent education: platform construction and curriculum development," Journal of Intelligent Manufacturing, vol. 32, pp. 32-40, 2021.

[12] M. Marques, C. Agostinho, G. Zacharewicz, and R. JardimGonçalves, "Decentralized decision support for intelligent manufacturing in Industry 4.0," Journal of Ambient Intelligence and Smart Environments, vol. 9, no. 3, pp. 299-313, 2017.

[13] V. K. Annanth, M. Abinash, and L. B. Rao, "Intelligent manufacturing in the context of industry 4.0: a case study of siemens industry[J]," Journal of Physics: Conference Series, vol. 1969, no. 1, Article ID 012019, 2021.

[14] J. Hu and S. Gao, "Research and application of capability maturity model for Chinese intelligent manufacturing," Procedia CIRP, vol. 83, pp. 794-799, 2019.

[15] B. Wang, F. Tao, X. Fang, C. Liu, Y. Liu, and T. Freiheit, "Smart manufacturing and intelligent manufacturing: a comparative review," Engineering, vol. 7, no. 6, pp. 738-757, 2021.

[16] W. Tao, Z.-H. Lai, M. C. Leu, Z. Yin, and R. Qin, “A self-aware and active-guiding training \& assistant system for workercentered intelligent manufacturing," Manufacturing letters, vol. 21, pp. 45-49, 2019.

[17] L. Wang, X. Chen, and Q. Liu, "A lightweight intelligent manufacturing system based on cloud computing for plate production," Mobile Networks and Applications, vol. 22, no. 6, pp. 1170-1181, 2017.

[18] M. N. Kripak, E. S. Palkina, and Y. A. Seliverstov, "Analytical support for effective functioning of intelligent manufacturing and transport systems," Materials Science and Engineering, vol. 709, no. 3, Article ID 033065, 2020.

[19] H. Yan, J. Yang, and J. Wan, "KnowIME: a system to construct a knowledge graph for intelligent manufacturing equipment," IEEE Access, vol. 8, pp. 41805-41813, 2020. 
[20] P. Fengque, T. Yifei, H. E. Fei, and L. Dongbo, "Research on design of the smart factory for forging enterprise in the industry 4.0 environment[J]," Mechanics, vol. 23, no. 1, pp. 146-152, 2017.

[21] Y. Tao, G. Zhao, Q. Li, and W. Zhao, "Reflections on facilitating the development of "Internet Plus" intelligent manufacturing in China," in Proceedings of the 2018 5th International Conference on Industrial Engineering and Applications (ICIEA), pp. 150-157, Singapore, April 2018. 